\title{
Left Lung, Superior Lobe, Apicoposterior Segment
}

National Cancer Institute

\section{Source}

National Cancer Institute. Left Lung, Superior Lobe, Apicoposterior Segment. NCI

Thesaurus. Code C132399.

The apicoposterior segment of the superior lobe of the left lung. 